APPENDICE IV B

\title{
DESCRIPTION D'UN CHOANOTAENIA PARASITE DE CERTHIA FAMILIARIS L. 1758
}

\section{Par Robert-Ph. DOLLFUS}

Matériel examiné : Trois individus à maturité chez un Certhia familiaris L. 1758, Richelieu (Indre-et-Loire), 4-8-1956.

Description : Longueur environ $20 \mathrm{~mm}$., plus grande largeur 1,15 mm. Le strobile est très fragile et se fragmente facilement, de sorte que la longueur d'un seul individu n'a pas pu être exactement mesurée. Pour deux individus, on a, en $\mu$ :

\begin{tabular}{|c|c|c|}
\hline Longueur du scolex. & 205 & 116 \\
\hline Largeur du scolex $\ldots \ldots \ldots \ldots \ldots \ldots$ & 220 & 124 \\
\hline Diamètre du rostellum $\ldots \ldots \ldots \ldots \ldots$ & 45 & 30 \\
\hline Diamètre des ventouses $\ldots \ldots \ldots \ldots \ldots$ & $103 \times 75$ & 50 \\
\hline Largeur du début du strobile & 255 & 103 \\
\hline
\end{tabular}

Les crochets du rostellum sont sur deux rangs, au nombre d'environ 18 , et sont longs, en moyenne, de $15 \mu$ (j'ai mesuré $14,15 \mu, 15,15 \mu, 16,5 \mu$, $17 \mu$ ), il est possible que les crochets du rang antérieur soient un peu plus longs que ceux du rang postérieur.

La segmentation débute tout près du scolex, par exemple à une distance de $50 \mu$; les proglottis sont d'abord plus larges que longs, rectangulaires, puis deviennent trapézoïdaux et un peu craspédotes, tantôt plus longs que larges, tantôt aussi longs que larges et à peu près carrés; il arrive qu'ils restent, même gravides, un peu plus larges que longs. Les dimensions de proglottis mûrs sont, par exemple, en $\mu: 738 \times 438,751 \times 860,769 \times 584$, $1.000 \times 1.060,1.075 \times 1.152$; des proglottis gravides, carrés, mesurent $922 \times 922 \mu$.

Les pores génitaux sont très irrégulièrement alternes et situés vers la fin du premier quart de la longueur. La poche du cirre, ovale, plus ou moins allongée, peut mesurer, par exemple, 103,7 $\times 47,4,128 \times 36,2,150 \times 45 \mu$; 


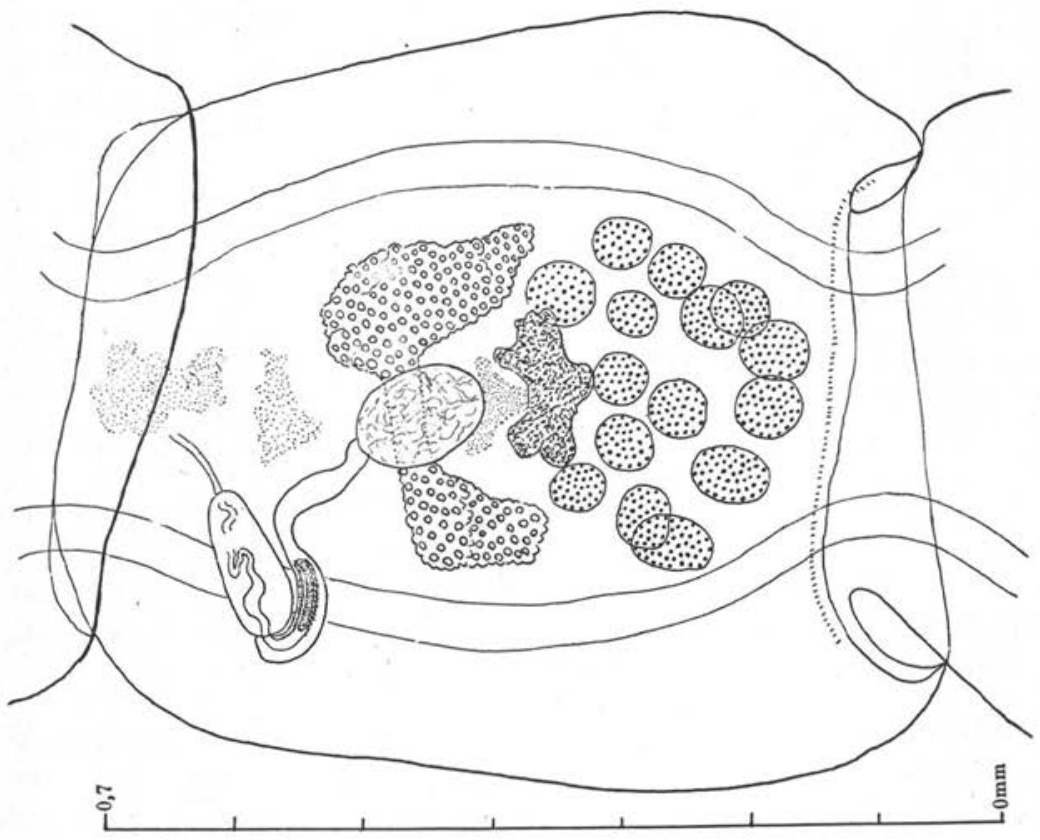

空
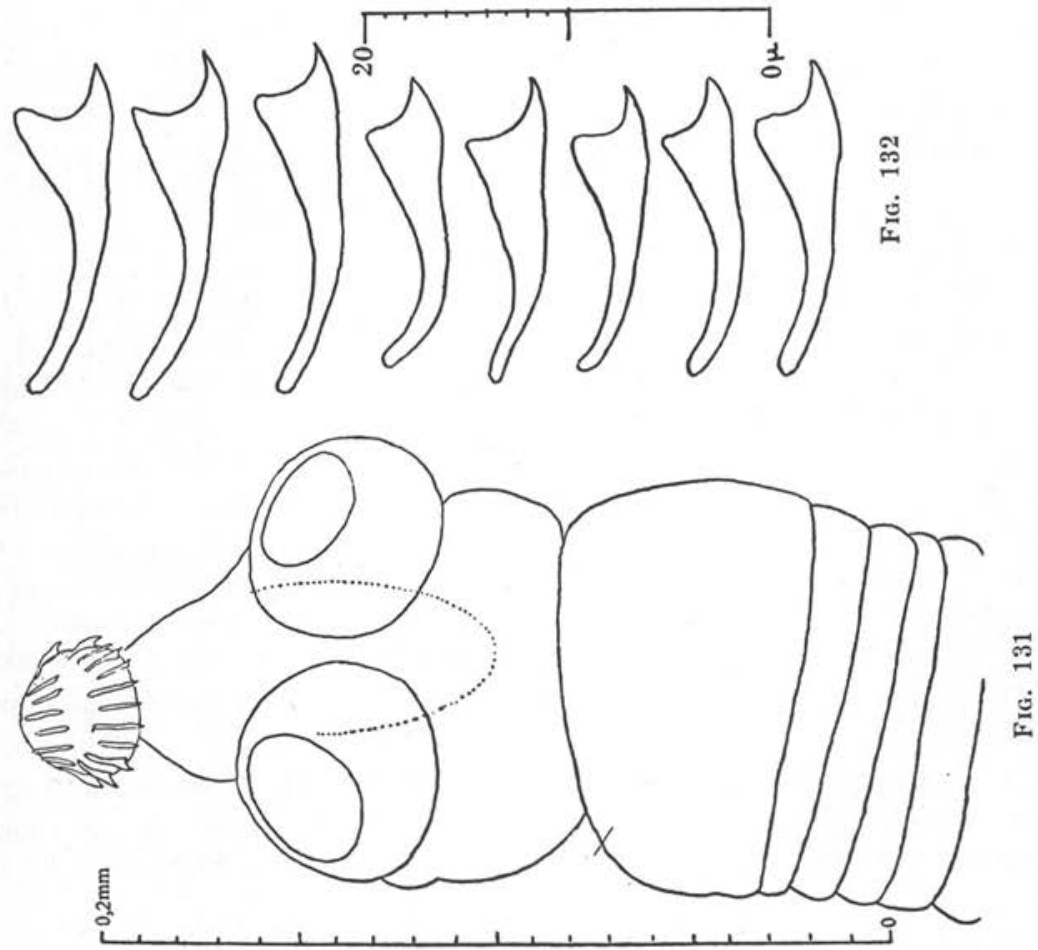


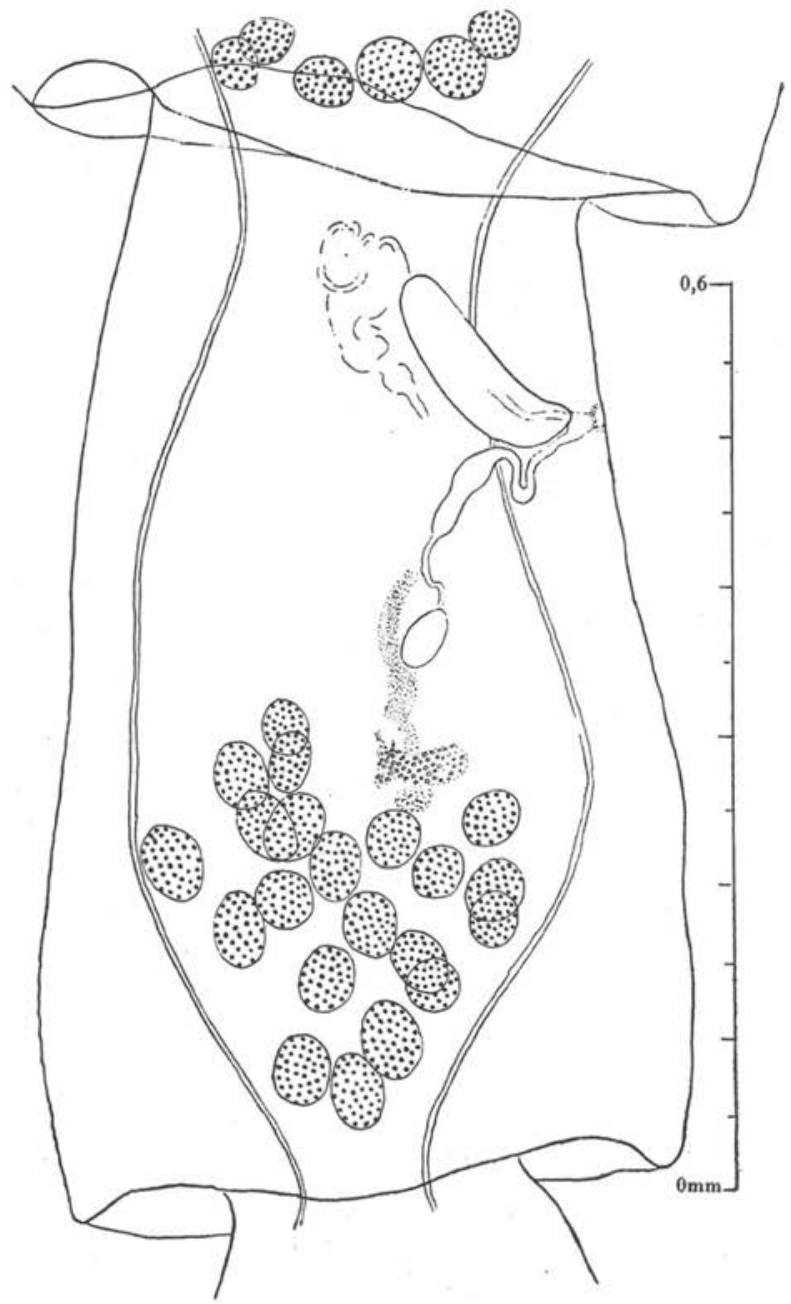

FIG. 133

FIG. 131-135. - Ghoanotaenia sp., intestin de Certhia familiaris L. 1758. Richelieu, 4-8-1956.

131, scolex et début du strobile, R.-Ph. D. delin. ; 132 , crochets du même scolex, R.-Ph. D. delin. ; 133, proglottis à maturité avec ovaire peu développé, R.-Ph. D. delin. : 134, proglottis à maturité avec cirre ayant pénétré dans le vagin, R.-Ph. D. delin. : 135, deux proglottis à maturité d'un autre individu. St. Deblock delin. 


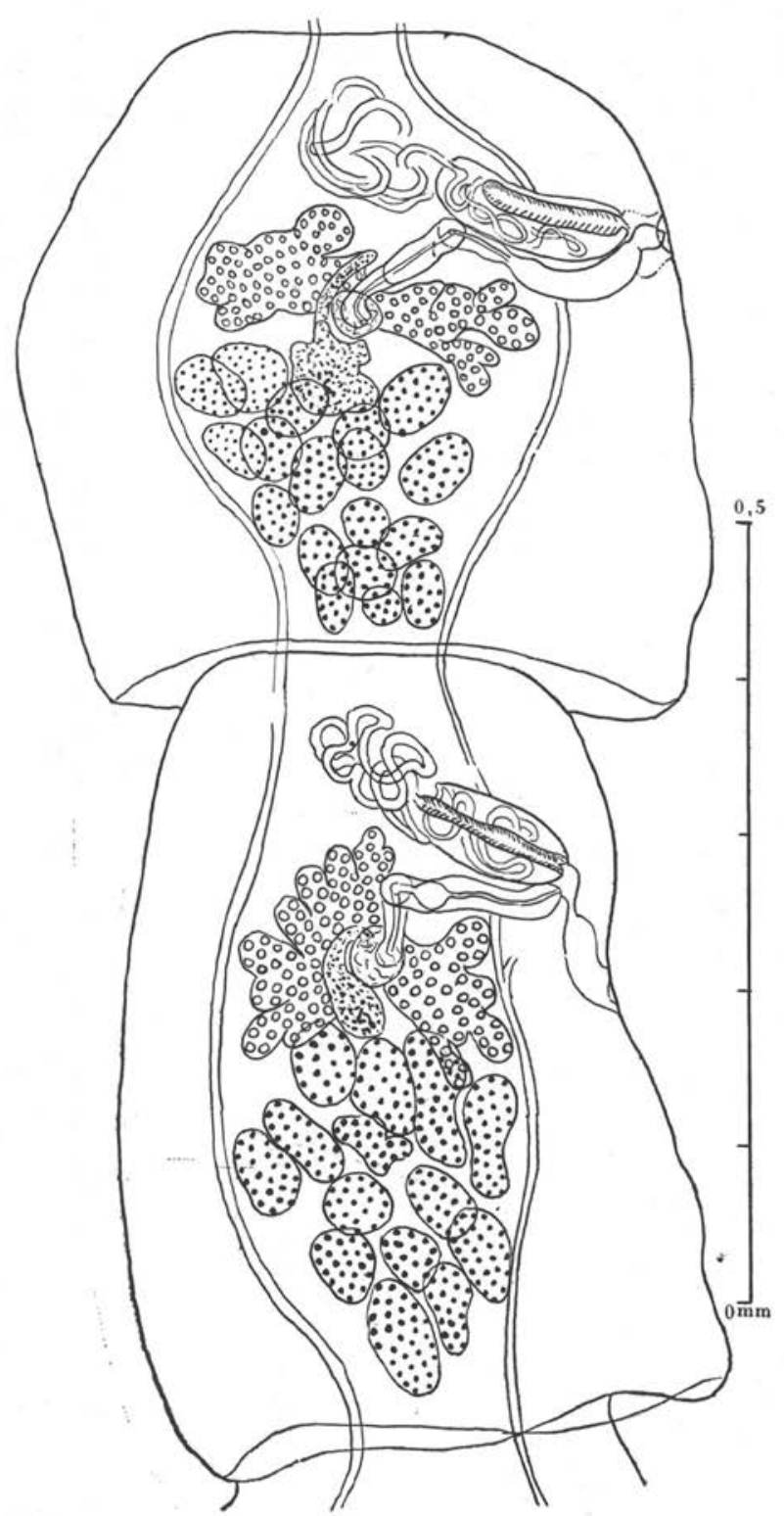

FIG. 135 
elle est située un peu obliquement et passe, avec le vagin, entre les deux canaux excréteurs longitudinaux. Le cirre est finement spinulé et grêle. Il y a de 12 à 20 testicules, plus ou moins ellipsoïdaux, et mesurant, en moyenne, $50 \times 40 \mu$, ils sont situés dans la moitié ou le tiers postérieur du segment, entre le niveau de l'ovaire et le bord postérieur du segment.

L'ovaire, médian, situé vers la mi-longueur du proglottis ou un peu en arrière, est de dimensions très variables, tantôt très petit, tantôt aussi large que la moitié de la largeur du proglottis et fortement lobé. Le vitellogène est très petit et situé immédiatement en arrière de l'ovaire ou à une petite distance; très souvent, il n'est pas bien discernable.

Le vagin est plus ou moins dilaté dans sa partie moyenne, il aboutit à un gros réceptaculum seminis ovale, mesurant, par exemple, $45 \times 22,50 \times$ $35,105 \times 80 \mu$, situé soit au niveau de l'isthme ovarien, soit plus en avant.

Dans beaucoup de proglottis, on observe l'autofécondation : le cirre a pénétré dans le vagin.

La formation de l'utérus n'est pas clairement observable, on voit tout de suite des capsules ovifères à un seul œuf dispersées dans le parenchyme. Il n'y a pas un utérus sacciforme persistant comme chez les Anomotaenia.

Les capsules ovifères, à paroi très mince, mesurent, par exemple, de $50 \times 55 \mu$ à $53,3 \times 66,6$ et contiennent chacune un œuf mesurant, au minimum, environ $35 \mu$ ou $30 \times 40 \mu$. Les oncosphères ont un diamètre minimum de 25 à $30 \mu$ et maximum de $40 \times 34,6$, avec des crochets d'environ $10 \mu$.

Nous ne connaissons pas de Choanotaenia de Passériforme paléarctique qui corresponde exactement à la description ci-dessus. 